\title{
Motion coherence and conjunction search: Implications for guided search theory
}

\author{
JON DRIVER \\ University of Cambridge, Cambridge, England \\ PETER MCLEOD \\ University of Oxford, Oxford, England \\ and \\ ZOLTAN DIENES \\ University of Sussex, Brighton, England
}

\begin{abstract}
Feature integration theory has recently been revised with two proposals that visual conjunction search can be parallel under some circumstances-either because items with nontarget features are inhibited, or because items with target features are excited. We examined whether excitatory or inhibitory guidance controlled conjunction search for an X oscillating in one direction among $\mathrm{Os}$ oscillating in that direction and $\mathrm{Xs}$ oscillating in another. Search was affected by whether items oscillated in phase with each other, and it was exceptionally difficult when items with target motion moved out of phase with each other and items with nontarget motion moved out of phase. The results suggest that conjunction search can be guided both by excitation of target features and by inhibition of nontarget features.
\end{abstract}

A wide range of evidence from neuroanatomy, neurophysiology, neuropsychology, and psychophysics has established that the primate visual system is modular (see, e.g., Braddick, Campbell, \& Atkinson, 1978; Cowey, 1985; Livingstone \& Hubel, 1987). If different visual attributes are indeed processed by relatively independent subsystems, how are these attributes combined to yield multidimensional percepts? Treisman's feature integration theory provides an attentional solution to this problem. Treisman and Gelade (1980) proposed that there are a number of visual modules, each extracting particular values (features) along a single visual dimension such as color, motion, or orientation. The modules provide "feature maps" that give the location of features on the dimension they code. These maps are derived in parallel, with features being encoded simultaneously at each location in the visual field. However, the coding of specific combinations of features is held to usually be a serial process. Feature maps from different modules can only communicate for one location at a time, under the control of spatial attention.

A wide range of results from the performance of human observers has been taken to support this view (Treisman, 1986, 1988). Perhaps the best known data come from visual search. The detection of the presence or ab-

\footnotetext{
This research was supported by a grant from the Medical Research Council (UK) to the first two authors, and a fellowship to the first author at the McDonnell-Pew Center for the Cognitive Neuroscience of Attention, University of Oregon. Correspondence may be addressed to Jon Driver, Department of Psychology, University of Cambridge, Downing Street, Cambridge CB2 3EB, England.
}

sence of a target defined by a single feature (e.g., a red item among green, or an $\mathrm{X}$ among $\mathrm{Os)}$ is often scarcely affected by the number of nontargets. This is expected on the basis of the theory, because the presence of a feature target should be indicated within a single feature map, and the information in these maps is derived in parallel. By contrast, search for a target defined by a conjunction of features (e.g., a red X among red Os and green Xs) can be linearly affected by the number of nontargets, with the slope of the increase in reaction time with set size being roughly twice as great for target-absent responses as it is for target detections (Treisman \& Gelade, 1980). This is consistent with the operation of a serial, self-terminating search. A serial search is expected, because finding a conjunction target requires information to be combined from separate feature maps, and according to Treisman (e.g., 1986), this combination usually only takes place at attended locations in a "master map." Conjunction search therefore requires that each item in the field be attended to in turn until the target is found.

The original version of feature integration theory has been challenged by several demonstrations of parallel conjunction search. Although Treisman and Gelade (1980) observed serial search for conjunctions of color and form, Nakayama and Silverman (1986) found parallel conjunction search for movement and binocular disparity, or color and binocular disparity. This initially suggested that depth might be a special case, consistent with the special role proposed for space in feature integration. However, McLeod, Driver, and Crisp (1988) found parallel search for conjunctions of movement and form, and Nakayama (reported in Treisman, 1988) found the same for conjunctions of 
movement and color. Moreover, Wolfe, Cave, and Franzel (1989) found parallel search for conjunction targets defined by combinations, of size, orientation, shape, and color. Thus, there are many counterexamples to the rule that conjunction search should be serial. Conjunction search for color and form has been found to be serial in some cases (Treisman \& Gelade, 1980) and parallel in others (Wolfe et al., 1989), so no complete account seems likely in terms of the particular dimensions that are involved.

Wolfe et al. (1989) and Treisman (1988; Treisman \& Sato, 1990) have both modified feature integration theory in an effort to accommodate parallel conjunction search, while retaining the idea of separate feature maps that communicate through a master map of locations. Treisman's (1988) key modification is that if a feature distinction is particularly salient, individual feature maps can inhibit nontarget locations on the master map so that they need not be attended during search. For example, if a color map inhibits the master map locations of green objects during search for a red $X$ among green $X s$ and red Os, the green Xs would be excluded from search. The subject would then effectively be looking for an X among Os (within the red stimuli), so parallel search should be found. Wolfe et al. (1989) made a similar proposal, which differs in that locations on the master map with target features are excited from the feature maps, rather than nontarget feature locations being inhibited. We will refer to the two proposals generically as the guided search account. In this paper, we seek to determine whether excitation of target features is used to guide conjunction search (Wolfe et al., 1989), or whether inhibition of nontarget features is the guidance mechanism (Treisman, 1988; Treisman \& Sato, 1990). Of course, it is possible that both are involved.

Previous data do not decide the issue conclusively. Wolfe et al. (1989) based their excitatory guidance proposal partly on the finding that search for triple conjunction targets differing from each distractor in two of their features was faster than search for the same targets among distractors that differed from them in only a single feature. This could be explained by guidance from two feature maps being more effective than guidance from one, whether this guidance is inhibitory or excitatory. Treisman and Sato (1990) performed an experiment that they interpreted as evidence for inhibitory rather than excitatory guidance. They found conjunction search to be harder among four different types of distractors than among two, even when the additional two distractor types were more discriminable from the target than those they replaced. They suggested that if guidance operated by excitation of target features alone, the addition of the more discriminable distractors could only help, because activation of the target features would be less likely to spread to the more discriminable distractors than to the original distractors that they replaced. By contrast, increasing the number of distractor types may make inhibitory guidance harder, because more nontarget features need to be inhibited.
However, the impairment that Treisman and Sato (1990) propose in the top-down inhibition of distractor features is by no means the only way to explain the detrimental effect of heterogeneity that they found (cf. Duncan \& Humphreys, 1989; Farmer \& Taylor, 1980). For example, bottom-up activation declines with increased interdistractor differences in Cave and Wolfe's (1990) excitatory computational model of guided search. Treisman and Sato (1990) concluded as follows:

We have presented an account based on inhibition rather than activation. Admittedly, the evidence distinguishing the two is still quite scanty, and an activation account may do equally well with most of the data. It is also quite possible that both play a role. (p. 477)

Given the equivocality of current data on the excitatory versus inhibitory guidance issue, we designed an experiment to examine whether the principle determinant of conjunction search efficiency is the ease with which a group of items with a shared nontarget feature can be rejected from search (inhibitory guidance), or the ease with which a group of stimuli sharing a target feature can be selected for search (excitatory guidance).

We exploited a phenomenon observed in pilot experiments on search for conjunctions of movement and form. The target was an $\mathrm{X}$ oscillating vertically on a screen among Os oscillating vertically and Xs oscillating horizontally. In pilot studies, we noted that this task was easy when all the moving stimuli were in phase with each other (i.e., in the vertical direction all stimuli moved up together and then down together, while in the horizontal direction all stimuli moved left together and then right together). But when items in both groups were out of phase with other items in their group, search became exceptionally hard. Thus, the coherence of the oscillations dramatically affected search difficulty. According to the inhibitory guidance account, this effect should be primarily due to the degree of motion coherence within the horizontal items (i.e., those with the nontarget motion). According to this account, inhibition of items with nontarget motion would become more difficult when the horizontally moving stimuli were out of phase. By contrast, according to the excitatory guidance account, the cost of incoherent motion should be attributable to the vertically moving items (i.e., those with the target motion). Excitation of items with target motion would become harder when the vertically moving items were out of phase. To decide whether excitatory or inhibitory guidance was involved, we varied the coherence of the target (vertical) motion and nontarget (horizontal) motion independently, to determine whether one was the crucial factor or whether both determined conjunction search efficiency.

The same form distinction ( $\mathrm{X}$ vs. $\mathrm{O}$ ) was required in all conditions, and thus, according to either version of guided search theory, any differences between the conditions should be attributable to differences in the efficiency of guidance by motion. The central tenet of both 
the excitatory and the inhibitory formulations is that guidance proceeds independently along different dimensions such as motion and form. Treisman and Sato (1990) provided some empirical support for this assumption, finding that each dimension contributed additively to conjunction search rates.

\section{METHOD}

\section{Subjects}

The twenty-four subjects, who were between 18 and 45 years of age, were paid volunteers from the Oxford University subject panel. All had normal or corrected acuity by self-report.

\section{The Task}

The subjects faced displays like those in Figure 1. The screen was rotated $45^{\circ}$ counterclockwise about an axis from the subject to the screen, so that stimuli oscillating vertically relative to the screen actually moved up-left and down-right relative to the subject, and stimuli oscillating horizontally relative to the screen actually moved up-right and down-left relative to the subject. This was to preempt an illusion reported by Driver, McLeod, and Dienes (in press), whereby stimuli moving vertically appear to travel faster than those moving horizontally at the same objective speed. For ease of exposition, stimuli will be described relative to the screen coordinates. Thus, those moving up-left and down-right relative to the subject will be called vertical, those moving up-right and down-left will be called horizontal.

In all conditions, the target was a vertically oscillating $X$, present on $50 \%$ of trials. Subjects responded "yes" (target present) with a button in one hand or "no" (target absent) with one held in the other. Half the subjects responded "yes" with the right hand and half with the left. On target trials, there were equal numbers of vertically oscillating $O s$ and horizontally oscillating $X s$, plus one vertically oscillating $X$. On nontarget trials, the vertically oscillating $X$ was replaced with an extra vertically oscillating $O$.

\section{The Display}

The screen was divided into an imaginary $21 \times 21$ grid. Os and $X s$ were distributed across the screen, each appearing in a cell from the imaginary grid, with the constraint that there be a maximum of one item per column, and one item per row in the imaginary grid. All stimuli moved at a speed of $2.1 \% / \mathrm{sec}$, and all reversed their direction of motion simultaneously every $100 \mathrm{msec}$, oscillating about the center of their cells in the imaginary grid. In cases of coherent oscillation, stimuli moved in phase (e.g., with coherent vertical motion, all vertical stimuli moved up together and then down together). In cases of incoherent oscillation, half the stimuli were $180^{\circ}$ out of phase with the other half. For example, with incoherent vertical oscillation, half the vertically oscillating stimuli moved up while the other half moved down. The display subtended $11^{\circ} \times 8^{\circ}$ at a viewing distance of $30 \mathrm{~cm}$. Individual letters subtended about $25^{\prime}$ of arc. The stimuli were displayed on an Electronic Visuals oscilloscope with a P34 phosphor, driven by a Cambridge Electronic Developments minicomputer. Each letter was drawn on a $9 \times 5$ dot matrix. Every $10 \mathrm{msec}$, the display was refreshed with, according to the direction of movement, either the vertical or the horizontal coordinates of the oscillating stimuli altered. Luminance levels of the screen and room were adjusted so that the oscillating stimuli left no apparent smear trail.

A trial started with a small fixation cross at the center of the screen for $500 \mathrm{msec}$, followed by the display until the subject responded. Finally, there was a pause of $1,500 \mathrm{msec}$ before the start of the next trial.

\section{Design}

A mixed design was employed, with set size $(9,15$, or 21 stimuli in a display) as the within-subject factor, and motion coherence providing four between-subject conditions. Six subjects were randomly assigned to each of the four groups. All subjects viewed displays in which there was one more item oscillating vertically than there was horizontally. For the all-coherent group of subjects, both vertical and horizontal items oscillated in phase. In the horizontalincoherent group, the vertically oscillating items were all in phase with each other, but the horizontally oscillating items were out of phase, so that half of them moved left while the remainder moved right. In the vertical-incoherent group, the vertically oscillating items were out of phase with each other, while the horizontally oscillating items were in phase. Finally, in the all-incoherent group, both vertical and horizontal stimuli oscillated out of phase. Typical displays for the four between-subject conditions are illustrated in Figure 1.

\section{Procedure}

On successive blocks of 50 trials, there were 9,15 , or 21 stimuli in the display. Each subject performed the search task at each of the three set sizes once in a random order. That order was then repeated three more times. The first two blocks at each set size were treated as practice. The data presented are the intersubject means of median reaction times (RTs) for correct responses on the last two blocks at each set size.

The subjects were given feedback on their median RT and error rate at the end of each block. They were directed to aim at an error rate between $5 \%$ and $10 \%$, and they were encouraged to speed up if they were below $5 \%$ and slow down if they were above $10 \%$. Three subjects were replaced due to high error rates.

\section{RESULTS}

Table 1 gives the mean results for each group of subjects; it includes the mean regression slopes for RT against set size in the four between-subject conditions. It can be seen that the all-incoherent condition was exceptionally difficult. It was by far the hardest, in terms of both search rate (as indicated by the slopes) and overall RT. For example, it took longer to detect a target in the 9-item displays of the all-incoherent condition than it did in the 21item displays from any other condition.

A three-way mixed analysis of variance (ANOVA) was performed on the "yes" RT data. Set size was the withinsubject factor, while horizontal coherence versus incoherence, and vertical coherence versus incoherence were the two between-subject factors. The ANOVA revealed main effects of vertical coherence versus incoherence $[F(1,20)=26.3, p<.001]$ and of horizontal coherence versus incoherence $\left.\left[F^{\prime} 1,20\right)=50.3, p<.001\right]$, plus an interaction between them $[F(1,20)=12.2, p<.01]$. Newman-Keuls tests indicated that overall RTs were greater in the all-incoherent condition than in any other ( $p<.01$ or better). RTs were also significantly longer in the horizontal-incoherent condition than in the allcoherent condition $(p<.05)$.

There was also a main effect of set size in the "yes" RT data $[F(1,20)=32.4, p<.001]$, which interacted with vertical coherence versus incoherence $[F(1,20)=$ 


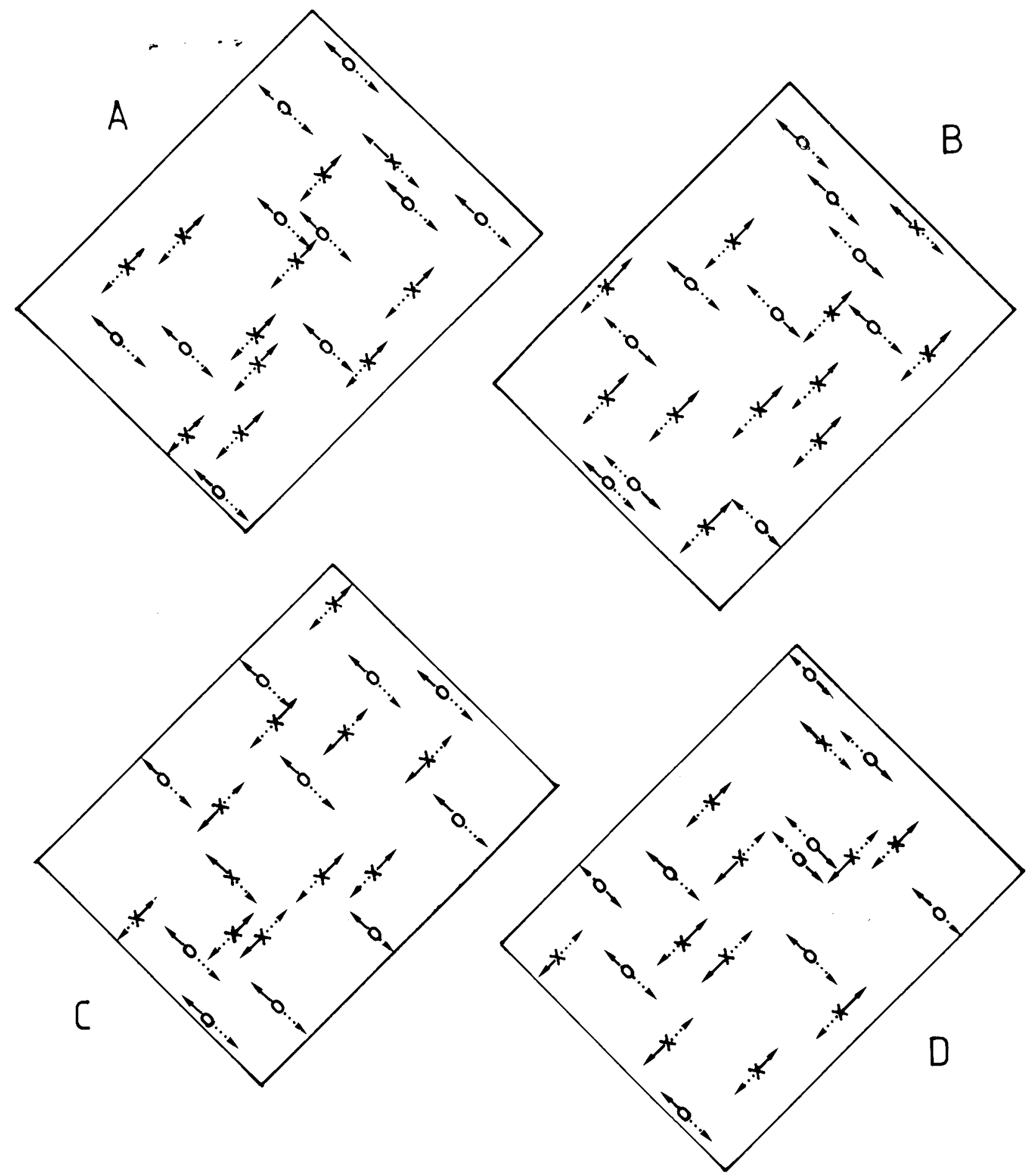

Figure 1. Examples of target-present displays at a set size of 21 from the four between-subject conditions. The screen was rotated $45^{\circ}$ counterclockwise about an axis from the subject to the screen, as depicted. Solid arrows depict the current direction of motion, dotted arrows the other direction in the oscillation. For convenience, the directions are referred to in screen coordinates (e.g., a stimulus moving "up" actually moved top-left relative to the subject). The target was a vertically oscillating $X$ among vertically oscillating $O$ s and horizontally oscillating Xs. (A) All coherent: vertically moving stimuli oscillate in phase, as do horizontally oscillating stimuli. (B) Vertical incoherent: horizontally moving stimuli oscillate in phase, but vertically moving stimuli oscillate out of phase (i.e., half of them are moving up while the remainder are moving down). (C) Horizontal incoherent: vertically moving stimuli oscillate in phase, but horizontally moving stimuli oscillate out of phase. (D) All incoherent: both vertically moving stimuli and horizontally moving stimuli oscillate out of phase. 
Table 1

Time (in Milliseconds) to Detect the Presence or Absence of a Vertically Oscillating $\mathbf{X}$ as a Function of Set Size

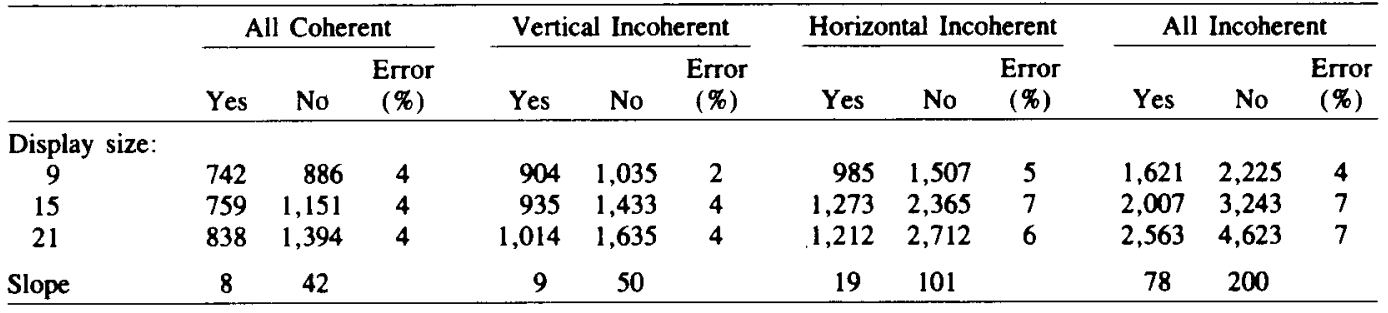

Note-Slope is given in milliseconds/item.

$10.5, p<.001]$, and horizontal coherence versus incoherence $[F(1,20)=16.3, p<.001]$. The three-way interaction was significant $[F(1,20)=10.0, p<.001]$, reflecting the exceptionally large set-size effect in the allincoherent condition.

Similar effects were observed in the "no" data. A threeway ANOVA revealed main effects of vertical coherence versus incoherence $[F(1,20)=12.7, p<.01]$ and horizontal coherence versus incoherence $[F(1,20)=60.7$, $p<.001]$, as well as an interaction between them $[F(1,20)$ $=5.8, p<.05]$. Newman-Keuls tests showed that the all-incoherent condition had longer RTs than did all the others $(p<.01)$. The horizontal-incoherent condition also differed from all the others $(p<.05)$. The effect of set size $[F(1,20)=101.3, p<.001]$ interacted with vertical coherence versus incoherence $[F(1,20)=8.3, p<$ $.01]$ and with horizontal coherence versus incoherence $[F(1,20)=28.4, p<.001]$. The three-way interaction again reached significance $[F(1,20)=7.2, p<.01]$, reflecting the exceptionally large set-size effect in the allincoherent condition.

Finally, the only significant effect in a three-way ANOVA on the error data was that of horizontal coherence versus incoherence $[F(1,20)=4.6, p<.05]$.

\section{DISCUSSION}

Our aim in this study was to examine whether the principal determinant of conjunction search efficiency is the ease with which a group of stimuli with a shared nontarget feature can be rejected from search (inhibitory guidance: Treisman, 1988; Treisman \& Sato, 1990), or the ease with which a group of stimuli sharing a target feature can be selected for search (excitatory guidance: Wolfe et al., 1989). In terms of guided search theory, the results demonstrate that both excitation and inhibition may be used to control search for a conjunction of movement and form.

Performance was affected by the coherence of motion in both the target direction (vertical) and the nontarget direction (horizontal). Search was relatively fast when there were two coherent motion groups, one of which had to be searched to find the target. If only the nontarget group moved coherently, search was still relatively easy; the guided search account would be that the coherent nontarget group was rejected from search via inhibitory guidance. When only the target group moved coherently, search was slightly slower, suggesting that in the present task it is harder to select a coherent group for search (by excitatory guidance) than to reject a coherent group from search (by inhibitory guidance). However, this effect was insubstantial relative to the dramatic disruption of search when both nontarget and target motion groups oscillated incoherently. If conjunction search had been controlled solely by inhibitory guidance, only the coherence of the nontarget motion should have determined search efficiency. Conversely, if excitatory guidance alone were in operation, only the coherence of the target motion should have affected search efficiency. However, the efficiency of conjunction search was determined jointly by the coherence of nontarget motion and that of target motion. This implies that both excitatory and inhibitory guidance can be used to control search for a conjunction of movement and form.

Duncan and Humphreys's (1989) similarity theory provides an alternative to guided search theory for understanding the efficiency of visual search, and stimulus heterogeneity plays a central role in their theory. Two factors are held by Duncan and Humphreys to influence the ease with which nontargets can be rejected and the target found: the similarity of the target to the nontargets, and the similarity (and hence grouping) of the nontargets to each other. These two factors interact, so that nontarget heterogeneity is most detrimental when target-nontarget similarity is high. Can our data be taken as an instance of this interaction?

Our data corroborate Duncan and Humphreys's (1989) emphasis on the importance of stimulus heterogeneity in visual search, and we do not consider our results inconsistent with their similarity theory. Nevertheless, we do not think that the theory makes a clear prediction for this experiment. Certainly, it would be a mistake to equate the observed interaction between the coherence of target (vertical) and nontarget (horizontal) motion with Duncan and Humphreys's predicted interaction between target-nontarget similarity and nontarget-nontarget similarity. In our study, making the horizontal motion incoherent presumably decreases the similarity of the horizontal nontargets to each other, while leaving target-nontarget similarity unaffected. Thus, the effects of horizontal incoherence could be attributed to decreased nontarget-nontarget similarity. 
However, mapping our manipulation of vertical motion onto Duncan and Humphreys's terminology is not as straightforward.-Vertical incoherence should reduce the similarity of the vertical nontargets to each other, but it is not entirely clear what the implications for target-nontarget similarity would be. At any one moment in a display with incoherent vertical motion, half the vertical items will be moving up while the others move down. The target, if present, will be in one of these two sets. Relative to coherent motion, incoherent vertical motion should therefore reduce target-nontarget similarity within a display for half the vertical items on target-present trials. From this perspective, vertical incoherence should, if anything, improve performance in terms of target-nontarget similarity! However, Duncan and Humphreys (1989) stressed that target-nontarget similarity is determined by matching between stimulus descriptions and an internal template for the target, rather than by similarities within the description for a given display. Thus, if we assume that the target template can specify vertical oscillation, rather than upward motion or downward motion in particular, vertical incoherence should not affect target-nontarget similarity. The observed effects of vertical incoherence would then have to be attributed to nontarget-nontarget similarity alone. Note that this argument assumes that upward and downward motion can be treated as equivalent for the purposes of template matching (target-nontarget similarity) but not for distractor grouping (nontarget-nontarget similarity). Similarity theory does not make strong predictions for our experiment, because such assumptions about the similarities involved are made after the results have been obtained.

The picture that emerges from our study is that conjunction search is relatively easy, provided that search can be restricted to a group of items that share one feature, within which the target is uniquely defined by its value on another dimension. In the present experiment, this can be achieved in two ways. First, attention may be restricted by excluding a coherent group of items with nontarget motion from search. This inhibitory guidance mechanism would operate successfully in the all-coherent and verticalincoherent conditions. Second, attention may be restricted by selecting for search a coherent group of items with the target motion. This excitatory guidance mechanism could operate in the all-coherent and horizontal-incoherent conditions. The slight cost observed for the horizontalincoherent condition relative to the all-coherent and vertical-incoherent conditions suggests that excitatory guidance is less efficient than inhibitory guidance in the present task. However, excitatory guidance clearly helps, because the efficiency of search breaks down dramatically in the all-incoherent condition, when neither excitatory nor inhibitory guidance can be efficient because target and nontarget motion are both incoherent.

The inferred difficulty in guiding conjunction search by exciting or inhibiting vertical or horizontal items that have out-of-phase motion suggests that the visual system cannot readily code these stimuli as moving either vertically or horizontally, as it tends to divide vertical motion into up or down, and horizontal motion into left or right. This is consistent with observations suggesting opponent coding of direction in the primate visual system. For example, single-cell recording reveals units with an excitatory response in the preferred direction and an inhibitory response in the opposite direction (Benevento, Creutzfeldt, \& Kuhn, 1972). Moreover, psychophysical studies of aftereffects including the well-known waterfall illusion suggest opponent coding of direction in human observers (e.g., Sekuler \& Ganz, 1963). Such opponent coding would make it particularly difficult to excite or inhibit the representation of items moving in opposite directions (e.g., up and down) relative to items moving in orthogonal directions (e.g., left and right). For example, modulating the activity of hypothetical "up" detestors would tend to produce an opposing modulation in "down" detectors, so that the net vertical activity would not differ from activity in left and right detectors as a result of the modulation. These considerations explain why both excitatory and inhibitory guidance would be ineffective in the allincoherent condition, in which search was exceptionally slow and effortful.

\section{REFERENCES}

Benevento, L. A., Creutzfeldt, O. D., Kuhn, U. (1972). Significance of intra-cortical inhibition in visual cortex. Nature, 238, 124-126.

Braddick, O., Campbell, F. W., Atkinson, J. (1978). Channels in vision: Basic aspects. In R. Held, H. W. Leibowitz, \& H.-L. Teuber (Eds.), Kandbook of sensory physiology (Vol. 8, pp. 3-38). New York: Pergamon.

CAve, K. R., \& Wolfe, J. M. (1990). Modelling the role of parallel processing in visual search. Cognitive Psychology, 22, 255-271.

CowEY, A. (1985). Aspects of cortical organization related to selective attention and selective impairments of visual attention. In M. I. Posner \& O. S. M. Marin (Eds.), Attention and performance XI (pp. 41-62). Hillsdale, NJ: Erlbaum.

Driver, J., McLeod, P., Dienes, Z (in press). Are direction and speed coded independently by the visual system?: Evidence from visual search. Spatial Vision.

DunCAN, J., \& HumphreYs, G. W. (1989). Visual search and stimulus similarity. Psychological Review, 96, 433-458.

FARMER, E. W., \& TAYLOR, R. M. (1980). Visual search through color displays: Effects of target-background similarity and background uniformity. Perception \& Psychophysics, 27, 267-272.

Livingstone, M., Hubel, D. (1987). Psychophysical evidence for separate channels for the perception of form, color, movement and depth. Journal of Neuroscience, 7, 3416-3468.

McLeod, P., Driver, J., \& CrISP, J. (1988). Visual search for a conjunction of movement and form is parallel. Nature, 332, 154-155.

NAkAYAMA, K., Silverman, G. (1986). Serial and parallel processing of visual feature conjunctions. Nature, 320, 264-265.

SeKULER, R., \&ANZ, L. (1963). A new aftereffect of seen movement with a stabilized retinal image. Science, 139, 419-420.

Treisman, A. (1986). Features and objects in visual processing. Scientific American, 255, 106-115.

Treisman, A. (1988). Features and objects: The 14th Bartlett Memorial Lecture. Quarterly Joumal of Experimental Psychology, 40A, 201-237. 
Treisman, A., \& Gelade, G. (1980). A feature integration theory of attention. Cognitive Psychology, 12, 97-136.

Treisman, A., SATo, S. (1990). Conjunction search revisited. Journal of Experimental Psychology: Human Perception \& Performance, 16, 459-478.

Wolfe, J. M., CAVe, K. R., \& Franzel, S. L. (1989), Guided search:
An alternative to the feature integration model for visual search. Journal of Experimental Psychology: Human Perception \& Performance, $15,419-433$.

(Manuscript received January 22, 1991; revision accepted for publication August 22, 1991.) 